\title{
Temporally Graded Retrograde Amnesia Following Separate and Combined Lesions of the Perirhinal Cortex and Fornix in the Rat
}

\author{
Kjesten A. Wiig, ${ }^{1,2}$ Leon N. Cooper, ${ }^{1,3}$ and Mark F. Bear ${ }^{4,5}$ \\ ${ }^{1}$ Institute for Brain and Neural Systems and Departments of ${ }^{2}$ Neuroscience and ${ }^{3}$ Physics \\ ${ }^{4}$ Howard Hughes Medical Institute \\ Brown University \\ Providence, Rhode Island 02912
}

\section{Abstract}

The involvement of the perirhinal cortex and the fornix in retrograde and anterograde amnesia in the rat was investigated in this experiment. Male Sprague-Dawley rats were trained on a series of five visual discrimination problems at distinct time intervals prior to receiving bilateral, electrolytic lesions of the perirhinal cortex or the fornix, combined lesions of both these structures, or sham operations. Following recovery from surgery, rats were retested on the preoperatively learned discrimination problems, as well as learning a new discrimination and discrimination reversal. Results indicated that all animals with lesions exhibited temporally graded retrograde amnesia, whereby memories acquired in the recent past (1-3 weeks) were impaired, and memories acquired in the remote past ( $6-8$ weeks) were spared. There was no difference in the magnitude of retrograde amnesia between the three lesion groups. Animals in the perirhinal, fornix, and combined lesion groups were able to learn a new discrimination problem at a rate comparable to control rats; however, the animals with lesions were impaired at learning the discrimination reversal. The perirhinal, fornix, and combined lesion animals also exhibited a significantly faster forgetting rate over a 2-week retention interval than control rats. These results suggest that medial temporal structures including the perirhinal cortex and the fornix are involved in the consolidation of mnemonic information and that their involvement in this process occurs over a discrete period of time.

\section{Introduction}

Throughout the latter half of this century, there has been considerable interest in determining how memories are encoded and stored within the mammalian brain. Although the precise mechanisms and loci of information storage have remained elusive, some progress has been made toward identifying both the brain structures involved in memory consolidation and the time course or duration of this process.

Much of our knowledge regarding the organization and neural foundations of normal memory processes has been obtained from the study of the human amnesic syndrome. Patients who have suffered damage to the medial temporal region of the brain, including the hippocampus, amygdala, and surrounding parahippocampal cortical regions typically exhibit severe anterograde amnesia (an inability to form new memories) and a more variable retrograde amnesia (loss of memories acquired prior to the precipitating incident). The phenomenon of retrograde amnesia has had a particularly large impact on ideas regarding memory consolidation processes. Specifically, retrograde amnesia is often temporally graded, in that memories acquired close to the time of the amnesia-

\footnotetext{
${ }^{5}$ Corresponding author.
}

LEARNING \& MEMORY 3:313-325 (c) 1996 by Cold Spring Harbor Laboratory Press ISSN1072-0502/96 \$5.00

$$
\begin{array}{lllllllllllllll}
L & E & A & R & N & I & N & G & \underset{313}{\mathbf{Z}} & M & E & M & O & R & Y
\end{array}
$$


inducing incident are lost, whereas memories formed in the more distant past are spared. In humans, memories formed several years prior to the onset of amnesia can be disrupted (Squire et al. 1975; Corkin 1984). This observation suggests that memory is not fixed at the time of learning but, rather, changes and becomes more stable with the passage of time (McGaugh and Gold 1976).

The development of primate and rodent models of retrograde amnesia has helped to further delineate which medial temporal lobe structures are involved in memory consolidation processes. Much of this research has focused on the hippocampal formation, and despite differences in tasks and species, similar patterns of results have been observed across studies. In these experiments, animals were trained on a task at different time intervals prior to receiving bilateral ablations of the hippocampal formation (Squire and Spanis 1984; Salmon et al. 1987; Wincour 1990; Zola-Morgan and Squire 1990; Kim and Fanselow 1992; Cho et al. 1995; Kim et al. 1995) or entorhinal cortex (Cho et al. 1993, 1995; Cho and Kesner 1996). The results of these experiments indicated that animals retained information that had been acquired in the remote past and lost information that had been acquired more recently. The temporal gradient of the retrograde amnesia varied from 10 days (Wincour 1990) to several months (Salmon et al. 1987). Although it is generally accepted that these results cannot be used to determine the time course of memory consolidation, they do support the idea of a gradually changing memory trace, which is more susceptible to disruption in early phases of memory consolidation.

Although the involvement of the hippocampal formation in retrograde amnesia has been well characterized, little is known about the contribution of the surrounding medial temporal cortical regions. Recently, it has been demonstrated that the rhinal cortex appears to play a particularly important role in the retention of newly acquired information. It has, for example, been shown that in the monkey, surgical ablations encompassing either the perirhinal and parahippocampal cortices (Zola-Morgan et al. 1989; Suzuki et al. 1993), or the perirhinal and entorhinal cortices (Eacott et al. 1994; Meunier et al. 1993; Murray et al. 1996 ) result in profound memory deficits on postoperatively acquired delayed-non-match-to-sample (DNMS) and complex discrimination tasks. Similarly, combined lesions of the perirhinal and entorhinal cortices in the rat result in impaired per- formance on DNMS (Otto and Eichenbaum 1992; Mumby and Pinel 1994) and paired-associate tasks (Bunsey and Eichenbaum 1993).

Moreover, it has been suggested that the perirhinal cortex alone may be responsible for much of the mnemonic processing required for adequate performance on tasks such as the DNMS. It has been demonstrated, for example, that lesions restricted to the perirhinal cortex in the monkey result in a more severe memory impairment than lesions confined to the entorhinal cortex (Meunier et al. 1993). Similarly, bilateral ablations of the perirhinal cortex alone in the rat have been shown to significantly disrupt performance on postoperatively acquired DNMS memory tasks (Wiig and Bilkey 1994b, 1995).

Given the importance of the perirhinal cortex to the retention of newly acquired information, it is of considerable interest to determine the extent of retrograde amnesia exhibited by animals with lesions of this area. The following experiment was designed to compare the retrograde consequences of lesions of the perirhinal cortex and the fornix. Rats learned two-choice visual discrimination problems at five different time intervals prior to receiving lesions of the perirhinal cortex, fornix, or both these structures. Retention for the preoperatively learned discrimination problems was assessed immediately following recovery from surgery. In addition, the ability of animals with lesions to learn a new discrimination problem and reversal and retain this information over a 2 -week interval was assessed.

\section{Materials and Methods}

\author{
SUBJECTS
}

Twenty-four male Sprague-Dawley rats, weighing between 150 and 180 grams at the start of behavioral training, served as subjects in this experiment. Subjects were individually housed in wire-mesh cages and were maintained on a 12-hron, 12-hr-off light-dark cycle. Rats had free access to water but were food deprived to $85 \%$ of their free-feeding body weight.

\section{APPARATUS}

The apparatus used in this experiment consisted of a perspex runway measuring $60 \mathrm{~cm}$ in length and $20 \mathrm{~cm}$ in width and with walls $50 \mathrm{~cm}$ in

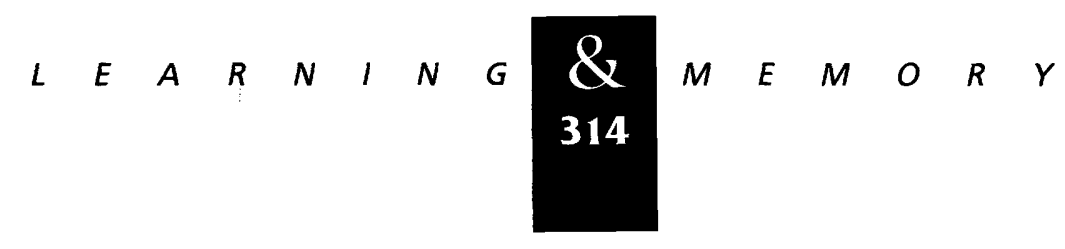


height (for a more detailed description of this apparatus, see Mumby et al. 1990). At each end of the runway were two identical goal areas, which were separated from the central portion of the maze by two opaque perspex guillotine doors. Within each goal area were two food wells, which were separated from each other by a short dividing wall. Food pellets were delivered to the wells via funnels that were mounted on the outside walls of the apparatus and connected to the food wells by means of plastic tubing.

A collection of 10 junk objects differing from one another in size, color, shape, and texture served as stimuli. The objects were large enough to cover a food well but were light enough to enable a rat to displace them. The food rewards were $45-\mathrm{mg}$ food pellets (BioServ).

\section{SURGERY}

All animals were anesthetized with sodium pentobarbital $(50 \mathrm{mg} / \mathrm{kg}$, i.p.) and placed in a stereotaxic apparatus, where a midline incision was made and the scalp retracted to expose the skull. The perirhinal cortex lesions were made by drilling holes through the skull at the coordinates: 3.3 , $4.3,5.3,6.3$, and $7.3 \mathrm{~mm}$ posterior to Bregma and $4.8 \mathrm{~mm}$ lateral to the midline. Monopolar electrodes (Teflon-coated wire, $125-\mu \mathrm{m}$ diam.), oriented laterally at $10^{\circ}$ from the vertical, were lowered at each site to a depth of $6.8 \mathrm{~mm}$ measured from the surface of the skull. DC current at $2 \mathrm{~mA}$ was passed through the electrodes for a duration of $10 \mathrm{sec}$. The electrodes were then removed, and the wound was sutured. Lesions of the fimbriafornix were made by placing electrolytic lesions bilaterally at the following three sites: $0.3 \mathrm{~mm}$ posterior to Bregma $(P), 0.4 \mathrm{~mm}$ lateral to the midline (L), and $4.2 \mathrm{~mm}$ ventral (V); $0.8 \mathrm{~mm} \mathrm{P,} 0.6 \mathrm{~mm} \mathrm{~L}$, and $6.3 \mathrm{~mm} \mathrm{~V} ; 1.3 \mathrm{~m} \mathrm{P}, 1.5 \mathrm{~m} \mathrm{~L}$, and $4.0 \mathrm{~m} \mathrm{~V}$. Combined lesions of the perirhinal cortex and fimbria-fornix were made in one operation, using identical surgical procedures to those just described. Control animals received sham operations in which holes were drilled in the skull overlying either the perirhinal cortex or the fimbria-fornix. Postoperatively, the animals were kept warm and monitored until spontaneous movement occurred. Once stabilized, they were returned to their home cages and left to recover for 7 days prior to behavioral testing.

\section{PROCEDURE}

PREOPERATINE TRAINING

The pretraining phase of the experiment consisted of six 15-min sessions during which the rats were habituated to the apparatus. For the first two sessions, each rat was placed into the apparatus, with both guillotine doors open, and allowed to explore for $15 \mathrm{~min}$. Pellets were scattered liberally throughout the length of the runway and in the food-well area. Food wells were rebaited after the pellets in each one had been consumed.

For the remaining four habituation session, the rats were trained to run from end to end of the apparatus. Each rat was initially placed in the center of the runway, with both guillotine doors closed. After $\sim 5 \mathrm{sec}$, one guillotine door was raised, allowing the rat access to the food wells. After the rat had eaten the pellets placed in both food wells and had begun to move toward the center of the runway, the closed guillotine door was raised. Once the rat had passed through this door, the far door was quietly lowered. Training continued in this manner until all rats had learned to approach the closed guillotine doors to gain access to the food wells on the other side of the door.

All rats were trained successively on five, twochoice object discrimination problems. For each of the five problems, two objects were presented to the rat, one of which was always associated with reinforcement $\left(\mathrm{S}^{+}\right)$and one of which was not $\left(\mathrm{S}^{-}\right)$. For each trial, the rat was placed in the center of the apparatus and the $\mathrm{S}^{+}$and $\mathrm{S}^{-}$positioned over two adjacent food wells at one end of the maze. The guillotine door in front of the objects was then raised, and the rat was allowed to approach the objects. The animal was rewarded with two food pellets for displacing the $\mathrm{S}^{+}$(correct response); if the $\mathrm{S}^{-}$was displaced (incorrect response), no reinforcement was delivered. Correction was allowed during the first discrimination session, during which the rat was allowed to displace the $\mathrm{S}^{+}$after making an error in order to obtain a food reward. Correction was not permitted for the remaining discrimination sessions. After the rat had finished eating the pellets and had returned to the center of the apparatus, the experimenter repositioned the $\mathrm{S}^{+}$and $\mathrm{S}^{-}$over the food wells at the opposite end of the maze in preparation for the next trial. Training continued in this manner (approximately five to seven daily training

$$
\text { ….... 娄 }
$$


Wiig et al.

sessions) until the rats had reached a criterion of 13 correct responses in two consecutive 16-trial daily training sessions. Training for the remaining four discrimination problems was conducted as described above, with a 1 week interval separating the acquisition of each problem. Thus, at five distinct time periods prior to surgery, the animals learned a different two-choice discrimination problem.

Immediately following acquisition of the final discrimination problem, the rats were divided into four performance-matched groups and received either sham operations $(n=6)$, electrolytic lesions of the fimbria-fornix $(n=6)$, perirhinal cortex $(n=6)$, or combined lesions of both these structures $(n=6)$.

\section{POSTOPERATIVE RETENTION TESTING}

Retention and reacquisition of the preoperatively acquired discrimination problems was assessed by a series of daily training sessions in which the five discrimination problems were represented to the rats. The rats were tested on two discrimination problems each day (eight trials per day for each problem), and testing continued until the animals had reacquired the preoperative criterion of 13 correct responses in 16 consecutive trials. The order of presentation was counterbalanced across both rats and groups, with half of the rats in each group being tested on different discrimination problems on each day of postoperative testing. Postoperative performance was measured by calculating the percentage of correct responses acquired on the first $\mathbf{1 6}$ trials. In addition, the number of trials taken to reattain criterion performance was determined.

Following completion of the postoperative retention tests, all rats were trained on a new twochoice discrimination problem in the same manner as described previously. Once the rats had attained criterion, the reward valence of the stimuli $(+$ or -$)$ was reversed and training continued until the rats had mastered the reversal problem. Two weeks later, retention for the discrimination reversal was assessed in a single session of 16 trials.

\section{HISTOLOGY}

On completion of the behavioral experiments, the animals were euthanized with an overdose of sodium pentobarbital and perfused through the heart with saline $(0.9 \%)$, followed by a $10 \%$ formalin solution. The brains were removed and immersed in a sugar formalin solution (30\%) until sectioning. Each brain was sectioned in the coronal plane ( $40-\mu \mathrm{m}$ steps), mounted, and stained with cresyl violet. Every second section was then analyzed to obtain a measure of lesion size for each hemisphere of each animal. Area measurements were conducted with the aid of a computer program that determined the total perirhinal cortical area and the total area of damaged tissue as outlined by the operator. An estimate of the total volume of damaged perirhinal cortical tissue was made by summing the values describing the lesion area and the values describing the cortical area and expressing the former as a percentage of the latter. In addition, coronal and lateral reconstructions of the lesions were made by plotting the extent of each ablation onto stereotaxic plates obtained from Paxinos and Watson (1986).

\section{Results}

\section{HISTOLOGICAL RESULTS}

PERIRHINAL CORTEX LESIONS

The perirhinal cortex was defined as the region of cortex that lies within the posterior half of the rhinal sulcus. The rostral portion of the perirhinal cortex is bordered by the posterior aspect of the insular cortex. The perirhinal cortex then continues caudally along the rhinal sulcus, with the most posterior aspect being found adjacent to the postrhinal cortex. The perirhinal cortex is bordered dorsally by the auditory cortex and ventrally by the entorhinal cortex (Deacon et al. 1983; Zilles 1990; Burwell and Amaral 1995).

Figure 1 illustrates the extent of the smallest and largest perirhinal cortical lesions. A substantial portion of the perirhinal cortex was destroyed in all six animals, with, on average, $62 \%$ of the perirhinal cortex being ablated bilaterally. Most lesions extended from the pia to the white matter of external capsule. In all cases, the lesions were confined to the perirhinal cortex; the surrounding entorhinal and temporal cortices and the hippocampal formation were undamaged.

FORNIX LESIONS

Figure 2 represents reconstructions of the smallest and largest fornix lesions. In five out of six

$$
\begin{array}{llllllllllllllll} 
& E & A & R & N & I & N & G & \begin{array}{l}
\boldsymbol{Q} \\
316
\end{array} & M & E & M & O & R & Y
\end{array}
$$



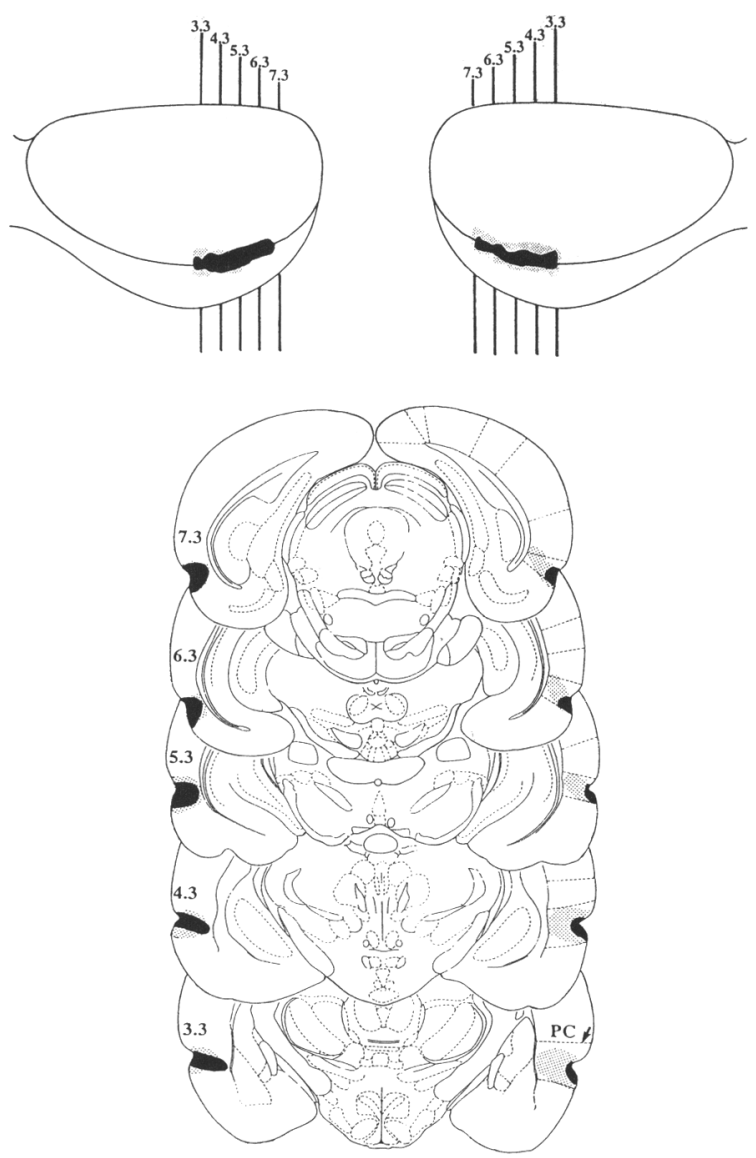

Figure 1: Reconstructions of the smallest (black) and largest (stippled) perirhinal cortex lesions plotted on a lateral view of the rat brain (top) and on successive coronal sections (bottom). Numbers represent the distance from Bregma. (PC) Perirhinal cortex.

animals, the dorsal fornix was completely severed, and three rats sustained damage to the descending columns of the fornix. The fimbria was damaged extensively in all six animals. In addition to the ablations of the fornix and fimbria, the anterior aspects of the lateral and triangular septal nuclei and the septofimbrial nucleus were damaged in all subjects. No damage to the hippocampus or to the thalamus was observed.

COMBINED PERIRHINAL FORNIX LESIONS

Figure 3 illustrates the extent of the smallest and largest combined perirhinal-fornix lesions. Histological examination revealed that three of the six combined lesion animals did not have a detectable perirhinal cortex lesion and, so, were discarded from subsequent behavioral analysis. In the three remaining rats, $\sim 65 \%$ of the perirhinal cortex was destroyed bilaterally. There was no evidence of damage to the entorhinal or temporal cortices or to the nearby hippocampal formation.

The dorsal fornix, descending columns of the fornix, and fimbria were all damaged extensively by the electrolytic lesion in these three subjects. As with animals in the fornix lesion group, additional damage to the lateral and triangular septal nuclei and to the septofimbrial nucleus was observed. There was no indication of damage to the thalamus or to the hippocampus.

\section{CORRELATIONS: LESION SIZE AND PERFORMANCE}

Correlation analyses were conducted to determine whether a relationship existed between lesion size and task performance, as measured by mean percent correct on reacquisition of discrimination 4. A moderately strong negative correlation between lesion size and performance was observed for the perirhinal lesion group $(r=-0.816$, $d f=4)$, fornix lesion group $(r=-0.804, d f=4)$,

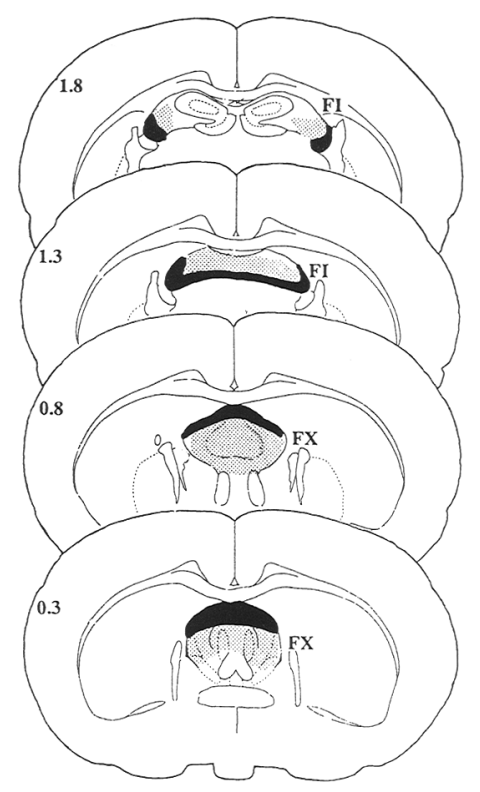

Figure 2: Successive coronal sections through the rat brain illustrating the location and extent of the smallest (black) and largest (stippled) fornix-fimbria lesion. Numbers represent the distance from Bregma. (FX) Fornix; (FI) fimbria.

\section{$L \begin{array}{llllllll}L & E & A & R & N & I & N & G\end{array}$




\section{Wiig et al.}
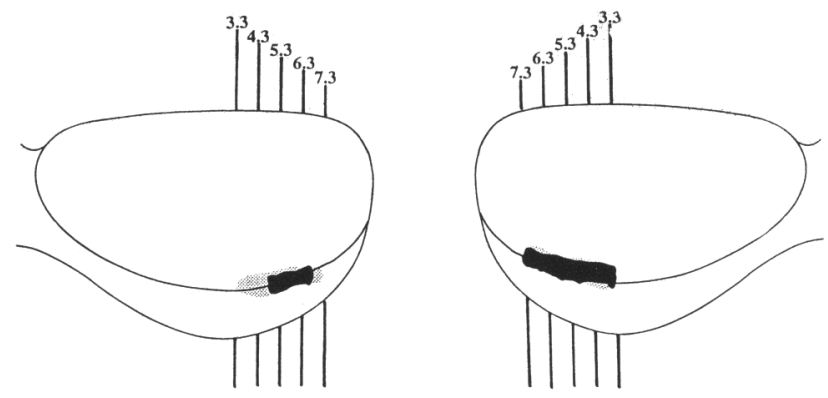

Figure 3: Reconstructions of the smallest (black) and largest (stippled) combined lesions plotted on a lateral view of the rat brain (top) and on successive coronal sections (bottom). Numbers represent the distance from Bregma. (PC) Perirhinal cortex;
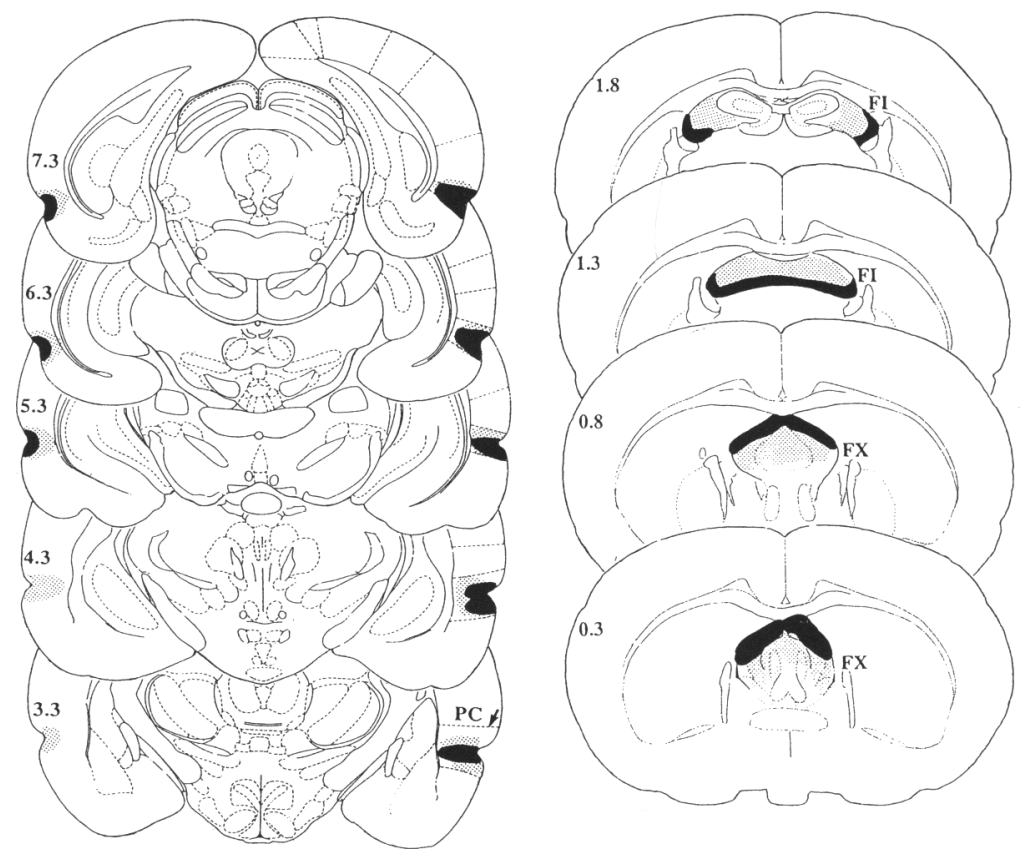
$(\mathrm{FX})$ fornix; (FI) fimbria.

and combined lesion group $(r=0.633, d f=1)$, suggesting that as lesion size increases, performance decreases (see Table $\mathbf{1}$ ).

\section{BEHAVIOR}

PREOPERATIVE PERFORMANCE

Animals required an average of 91.4 trials to learn the first discrimination problem (D1). Subsequent discrimination problems were learned more rapidly, with $\mathrm{D} 2, \mathrm{D} 3, \mathrm{D} 4$, and $\mathrm{D} 5$ requiring $71.4,32.0,74.6$, and 68.5 trials, respectively. Analysis of variance (ANOVA) revealed a significant difference in the number of trials required to learn the discrimination problems $[F(4,100)=42.14$, $P<0.0001$ ], and a Student-Newman-Keuls posthoc test demonstrated that discrimination problems presented later in the testing period (D4 and D5) were learned significantly faster than discrim- inations presented earlier in the testing series (DI and D2) $(P<0.05)$. D3 was learned significantly faster than any other discrimination problem $(P<0.05)$.

Table 2 presents discrimination scores (mean percent correct) obtained on the last day of testing for each discrimination problem. No significant difference between the performance of animals who subsequently received lesions of the perirhinal cortex, fornix, or combined lesions was observed.

\section{RETENTION OF PREOPERATIVELY LEARNED}

DISCRIMINATIONS

Figure 4 illustrates the results of the retention test given 1 week after surgery. The test measured retention of information that had been learned by the rats at different time periods (ranging from 1 to 8 weeks) prior to surgery. As can be seen from Figure 4, the forgetting patterns appear to 
Table 1: Estimated damage (in percentage of normal) and performance of each rat in perirhinal, fornix, and combined lesion groups

\begin{tabular}{|c|c|c|c|c|}
\hline Group & Rat & \multicolumn{2}{|c|}{ Lesion size } & Performance ${ }^{a}$ \\
\hline \multirow[t]{6}{*}{ Perirhinal } & 4 & \multicolumn{2}{|c|}{59} & 75.0 \\
\hline & 10 & \multicolumn{2}{|c|}{68} & 81.25 \\
\hline & 11 & \multicolumn{2}{|c|}{70} & 68.75 \\
\hline & 18 & \multicolumn{2}{|c|}{62} & 68.75 \\
\hline & 20 & \multicolumn{2}{|c|}{45} & 93.75 \\
\hline & 22 & \multicolumn{2}{|c|}{70} & 62.5 \\
\hline \multirow[t]{7}{*}{ Fornix } & 3 & \multicolumn{2}{|c|}{70} & 68.75 \\
\hline & 7 & \multicolumn{2}{|c|}{54} & 87.5 \\
\hline & 8 & \multicolumn{2}{|c|}{52} & 75.0 \\
\hline & 9 & \multicolumn{2}{|c|}{50} & 87.5 \\
\hline & 17 & \multicolumn{2}{|c|}{60} & 75.0 \\
\hline & 19 & \multicolumn{2}{|c|}{65} & 68.75 \\
\hline & & PC & $\mathrm{FF}$ & \\
\hline \multirow[t]{3}{*}{ Combined } & 6 & 59 & 65 & 87.5 \\
\hline & 12 & 70 & 75 & 75.0 \\
\hline & 24 & 68 & 70 & 50.0 \\
\hline
\end{tabular}

(PC) Perirhinal component of combined lesion; (FF) fornix-fimbria component of combined lesion.

apostoperative retention scores for discrimination 4.

vary between groups. This observation was confirmed by a repeated measures ANOVA, which revealed a significant group by time interaction $[F(12,68)=2.228, P<0.01]$. Post hoc analysis (Student-Newman-Keuls) demonstrated that the performance of all three lesion groups was significantly poorer than that of the control group for discrimination problems learned at 1 and 2 weeks prior to surgery $(P<0.05)$. The performance of the perirhinal and fornix lesion groups was found to be significantly impaired relative to that of the control group for D3, learned at 4 weeks prior to surgery $(P<0.05)$. No significant difference between the performance of control and experimental groups was observed for D2, which was learned 6 weeks prior to surgery. There was, however, a significant difference in performance for D1, learned at 8 weeks before surgery. Student-Newman-Keuls post hoc analysis demonstrated that the performance of the combined lesion and perirhinal lesion group was significantly poorer than that of control animals at this time point $(P<0.05)$.

The ANOVA also revealed a main effect of group $[F(3,17)=10.979, P<0.0003]$. Further analysis using a Student-Newman-Keuls post hoc test demonstrated that the control group performed significantly better than the lesion groups $(P<0.05)$; however, no significant difference between the performance of the perirhinal, fornix, or combined lesion groups was observed. In addition, a main effect of time was found $[F(4,68)=21.955$, $P<0.0001$ ), indicating that retention of information varied according to the time period in which it was learned.

The performance of control and experimental groups on the first set of eight trials and the second set of eight trials of testing for each discrimination problem was compared to assess whether a significant degree of relearning occurred within the early phases of the retention test. No significant difference between performance on the first and second set of eight trials was observed for any of the five discrimination problems $(P>0.05)$.

\section{POSTOPERATIVE LEARNING AND RETENTION}

Rats with lesions of the perirhinal cortex, fornix, or both these structures were able to learn a new discrimination problem at an identical rate to that of control animals; rats in each of the four groups required 32 trials to attain criterion on this problem. The lesion groups were, however, impaired in learning the reversed discrimination problem. Perirhinal, fornix, and combined lesion rats required significantly more trials to reach criterion in the reversal problem as compared with control rats $[F(3,20)=7.159, P<0.001]$. There was no significant difference between the performance of the three lesion groups on this measure (see Fig. 5A).

Table 3 and Figure 5B show retention of the reversed discrimination problem over a 15-day time interval. An ANOVA revealed a significant group $\times$ time interaction $[F(3,17)=6.152$, $P<0.005$ ], whereby the performance of the lesion groups decreased over time, whereas the performance of the control groups remained high. No other significant main effects were observed.

\section{Discussion}

EFFECTS OF LESIONS OF THE PERIRHINAL. CORTEX AND FORNIX ON MEMORY CONSOLIDATION

The results of the present study provide evidence that the perirhinal cortex and fornix are

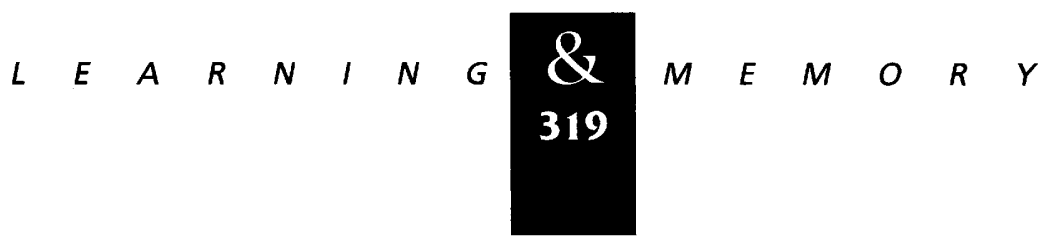


Table 2: Preoperative scores (mean percent correct, \pm S.E.M.) on the last day of testing for each of the five discrimination problems learned by animals in control and lesion groups

\begin{tabular}{lccccc}
\hline Group & 1 & 2 & 3 & 4 & 5 \\
\hline Control & $97.90 \pm 1.3$ & $93.75 \pm 2.2$ & $94.79 \pm 1.9$ & $94.79 \pm 1.9$ & $90.62 \pm 3.5$ \\
Perirhinal & $98.21 \pm 1.0$ & $95.83 \pm 3.0$ & $96.87 \pm 2.1$ & $89.58 \pm 3.1$ & $89.58 \pm 3.4$ \\
Fornix & $95.83 \pm 1.3$ & $96.87 \pm 2.1$ & $97.92 \pm 1.3$ & $91.25 \pm 3.8$ & $92.71 \pm 2.9$ \\
Combined & $90.62 \pm 2.6$ & $92.71 \pm 3.4$ & $93.75 \pm 2.2$ & $94.79 \pm 2.5$ & $94.79 \pm 1.9$ \\
\hline
\end{tabular}

involved in memory consolidation. In this experiment, rats with perirhinal cortex or fornix lesions exhibited temporally graded retrograde amnesia, whereby retention of discriminations learned between 1 and 4 weeks prior to surgery was poor, and retention of the discrimination problem learned at 6 weeks prior to surgery was comparable with that of control rats. Animals with combined lesions of both the perirhinal cortex and the fornix exhibited a similar retrograde amnesia to that of the perirhinal or fornix rats, showing poor memory for recently acquired information and better memory for remotely acquired information.

Interestingly, animals that had received lesions of either the perirhinal cortex or combined
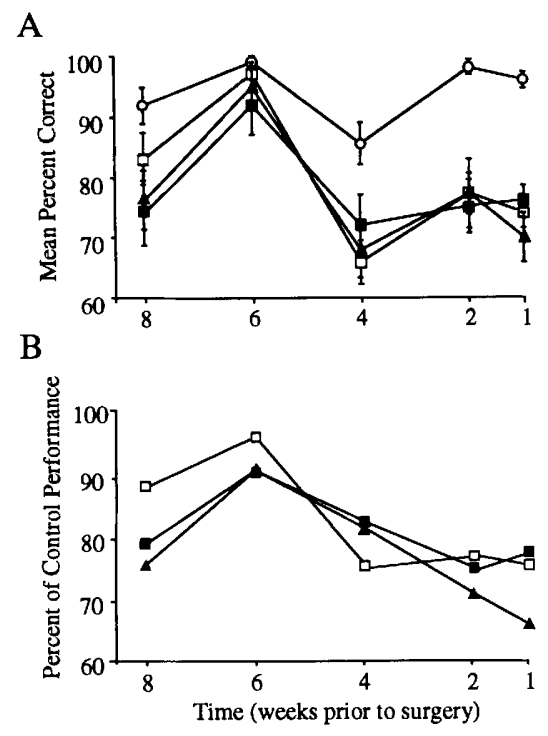

Figure 4: $(A)$ Postoperative retention (mean percent correct on the first 16 trials) of five different object pairs, learned at five different time intervals prior to surgery. Animals with perirhinal $(\boldsymbol{\square})$, fornix $(\square)$, and combined lesions $(\boldsymbol{\Delta})$ exhibited temporally graded retrograde amnesia as compared to control $(O)$ animals. Error bars depict S.E.M. (B) Performance of the three lesion groups expressed as percent of control performance. lesions of both the perirhinal cortex and fornix exhibited impaired performance relative to controls on D1, learned 8 weeks prior to surgery. Although it is unclear as to exactly why these two groups of animals were impaired on the retention of this discrimination, it is possible that the perirhinal cortex lesion resulted in an accelerated rate of forgetting of long-term memories. It is important to note, however, that the measure of retention used in the present experiment cannot be regarded as a pure indicator of memory, as more than one trial was required to reliably assess performance on the discrimination problems. Although a statistically significant degree of relearning between the first and second day of retention testing was not observed in the present experiment, it is still possible that the retention measure was confounded by reacquisition processes that may have been used by the rats during the initial trials of retention testing.

The finding of retrograde amnesia following damage to the medial temporal region is consistent with previous research conducted in humans, monkeys, and rats. Retrograde amnesia has, for example, been observed in rats following lesions of the hippocampus (Wincour 1990; Kim and Fanselow 1992; Cho et al. 1995; Kim et al. 1995) or entorhinal cortex (Cho et al. 1993; Cho and Kesner 1996). In addition, it has been demonstrated recently that rats with lesions of the perirhinal cortex exhibit retrograde amnesia for a simple brightness discrimination problem learned $24 \mathrm{hr}$ prior to surgery (Myhrer and Wangen 1996). Collectively, these results suggest that the medial temporal region of the brain, including the hippocampus, fornix, entorhinal cortex, and perirhinal cortex, play a necessary role in the development and maintenance of some types of mem. ory trace.

In addition to the retrograde amnesia observed in the present experiment, animals with lesions of the perirhinal cortex and/or fornix were

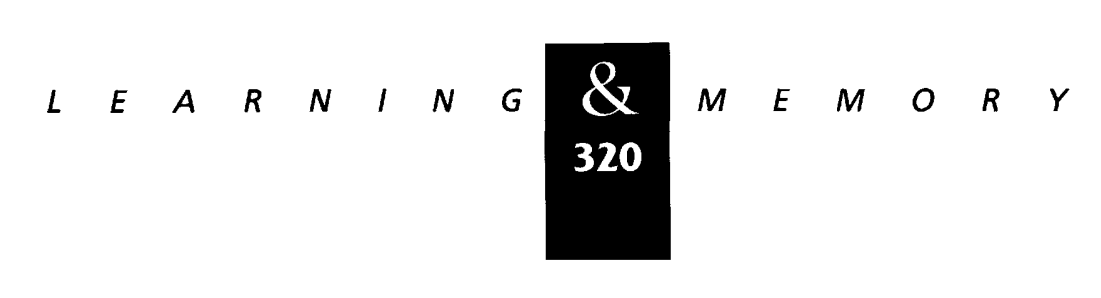


Table 3: Retention of discrimination reversal over a 15-day interval

\begin{tabular}{|c|c|c|c|c|}
\hline \multirow[b]{2}{*}{ Group } & \multirow{2}{*}{$\begin{array}{l}\text { Day } 0 \\
\text { (last day of training) } \\
\% \text { correct ( } \pm \text { S.E.M.) }\end{array}$} & \multicolumn{3}{|c|}{ Day 15} \\
\hline & & $\begin{array}{l}\% \text { of control } \\
\text { performance }\end{array}$ & $\%$ correct $( \pm$ S.E.M.) & $\begin{array}{l}\% \text { of control } \\
\text { performance }\end{array}$ \\
\hline Control & $86.45 \pm 2.9$ & & $94.7 \pm 1.0$ & \\
\hline Perirhinal & $89.58 \pm 2.6$ & 103.6 & $85.41 \pm 3.8$ & 90.2 \\
\hline Fornix & $89.58 \pm 2.6$ & 103.6 & $83.33 \pm 2.1$ & 88.0 \\
\hline Combined & $88.54 \pm 1.9$ & 102.4 & $82.29 \pm 3.7$ & 86.9 \\
\hline
\end{tabular}

impaired in the postoperative acquisition of a discrimination reversal problem. Behavioral observations made during the experiment suggested that animals with lesions were somewhat more likely to preserve the prior response strategy, rather than to treat the reversal as a new discrimination problem with new reward valences. This finding is

A
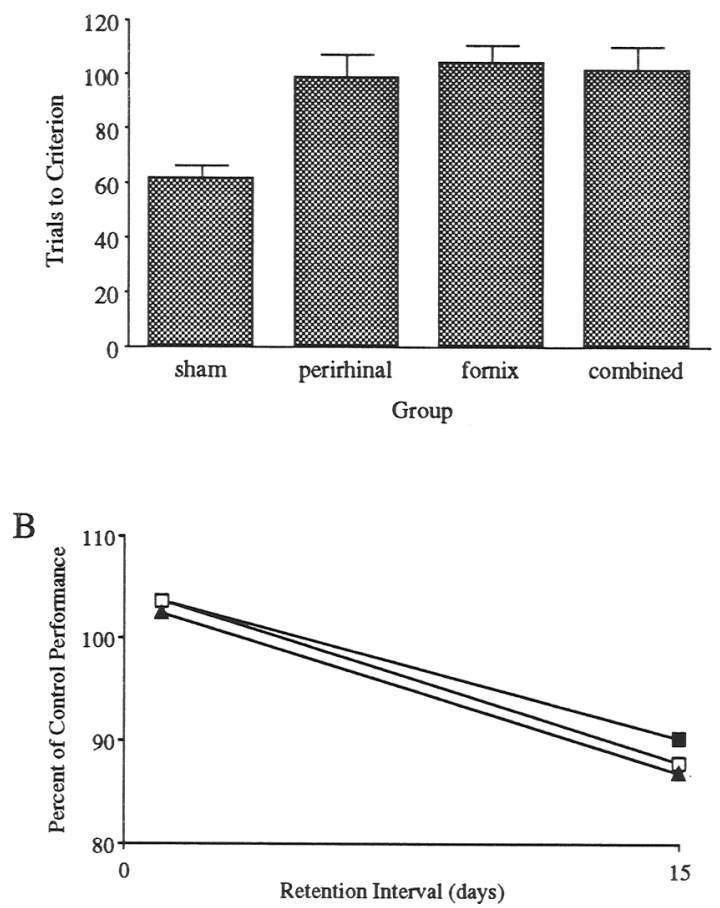

Figure 5: (A) Performance on the postoperatively learned discrimination reversal. Animals with perirhinal, fornix, and combined lesions required significantly more trials to reach criterion as compared with controls. Error bars depict S.E.M. (B) Postoperative retention of the discrimination reversal over a 15-day interval, expressed as a percentage of control (O) performance. Perirhinal $(\square)$, fornix $(\square)$, and combined lesion $(\boldsymbol{\Delta})$ groups exhibited an accelerated rate of forgetting. consistent with that of Cho et al. (1993), who also observed disrupted reversal acquisition in mice following entorhinal cortex ablations. These findings, however, stand in contrast to those of Eichenbaum et al. (1986), who demonstrated that rats with lesions of the fornix were able to learn an odor-guided discrimination reversal better than control animals. It is possible that differences in the experimental protocol (odor discrimination vs. visual discrimination) and lesion extent and location may account for these discrepancies.

If memory consolidation is a process entailing the maintenance and stabilization of the memory trace, then the absence of such a process should result in an abnormally rapid rate of forgetting. In the present experiment, animals with lesions of either the perirhinal cortex or fornix or combined lesions of both these structures exhibited an accelerated rate of forgetting over a 2 -week interval relative to control animals. This finding is consistent with previous research that demonstrated that rats with lesions of the hippocampus (Vnek et al. 1995) or entorhinal cortex (Staubli et al. 1986; Levisohn and Isacson 1991; Cho et al. 1993) were unable to maintain the integrity of information over long (up to 3 weeks) delay intervals. In addition, similar results have been obtained from studies conducted with the noted amnesic patient H.M. H.M. is able to perform normally on a visual recognition task providing the delay interval is 1 day or less. If the retention interval is extended to 1 week or more, H.M.'s performance is impaired severely relative to that of control subjects (Huppert and Piercy 1979; Squire 1981).

\section{MEDIAL TEMPORAL LESIONS, CONSOLIDATION, AND DECLARATIVE MEMORY FORMATION}

The results of the present experiment demonstrated that lesions of the perirhinal cortex and fornix did not have an additive effect. Lesions of

$$
\begin{array}{llllllll}
L & E & A & R & N & I & N & G \\
\mathbf{3 2 1} & \mathbf{Q} & M & E & M & O & R & Y
\end{array}
$$


either the perirhinal cortex or fornix or combined lesions of both structures resulted in an almost identical temporally graded retrograde amnesia. It could be argued, therefore, that the resultant retrograde memory deficit was simply a function of destroying brain tissue, rather than destroying tissue in a specific memory-related region. It should be noted, however, that not all lesions result in temporally graded retrograde amnesia. Lesions of the parietal cortex, for example, result in a relatively mild, ungraded retrograde amnesia (Cho and Kesner 1996), whereas lesions of the dorsomedial thalamic region in rats do not produce temporally graded retrograde amnesia (Wincour 1990). Taken together, these findings suggest that structures within the medial temporal region of the brain possess some characteristic that enables them to participate actively in the memory consolidation process. Furthermore, the fact that damage to any of the investigated structures within the medial temporal lobe results in a retrograde amnesia that is similar in terms of duration and severity suggests that the medial temporal region may operate as an integrated system in the consolidation of newly acquired information, and removing any one component of the system may be as devastating as removing more than one. Alternatively, these results could support the idea of a hippocampal-dependent memory consolidation system. Because the functional effect of both the perirhinal cortex and the fornix lesions is to disrupt hippocampal processing by means of deafferentation, the hippocampal formation itself could be viewed as the locus critical to memory consolidation.

It is plausible that structures within the medial temporal region of the brain may make substantially different contributions toward various facets of memory. The hippocampus, for example, does not appear to be essential for the acquisition and maintenance of nonspatial declarative information. Neither monkeys (Gaffan 1994) nor rats (Otto and Eichenbaum 1992) with lesions of the hippocampus or fornix are impaired severely on the DNMS task, a paradigm that is considered to tax declarative memory. In contrast, monkeys (Zola-Morgan et al. 1989; Meunier et al. 1993) or rats (Mumby and Pinel 1994; Wiig and Bilkey 1995) with ablations of the perirhinal, entorhinal, or parahippocampal cortices exhibit substantial impairments on DNMS tasks, suggesting that these areas are critically involved in the representation of declarative information over limited periods of time. In addition, rhinal cortical regions do not appear to play an essential role in the processing of spatial information (Gaffan 1994; Wiig and Bilkey 1994a), whereas the hippocampal formation does (Morris et al. 1982). Taken as a whole, these observations suggest that there may be some degree of functional independence among structures within the medial temporal system in terms of their involvement in the acquisition, retention, and consolidation of information.

\section{PUTATIVE MECHANISMS OF MEMORY CONSOLIDATION}

Although the direction of information flow into and out of the medial temporal system has yet to be determined, it is tempting to speculate that information in a given modality may enter the brain and be temporarily stored in the neocortical region that processes information for that modality. If the information is to be permanently stored, the neocortical region may activate the medial temporal system via reciprocal connections with, for example, the perirhinal or parahippocampal cortices. These cortical regions receive multimodal information from widespread neocortical areas and provide massive input to the entorhinal cortex and hippocampal formation (Deacon et al. 1983; Insausti et al. 1987). The perirhinal or entorhinal cortex or the hippocampus may initiate some process that ultimately results in the permanent storage of information in neocortex. Because the destruction of any one of these medial temporal regions produces a comparable retrograde amnesia, it is reasonable to assume that these structures may be equally involved in memory consolidation. Although the nature of this process is at present ambiguous, it may involve the activation of outflow pathways from the fornix to the septum and hypothalamus (Swanson and Cowan 1979), which, in turn, project back to multiple neocortical regions (Saper 1985).

Although the structures that participate in memory consolidation are now known with increased clarity, the neurobiological mechanism by which consolidation occurs has yet to be fully elucidated. There is, however, some evidence to suggest that the formation of long-term memories requires gene expression and protein biosynthesis (for review, see Davis and Squire 1984; Matthies 1989). There have, for example, been numerous reports demonstrating that agents that inhibit protein synthesis block the formation of long-term

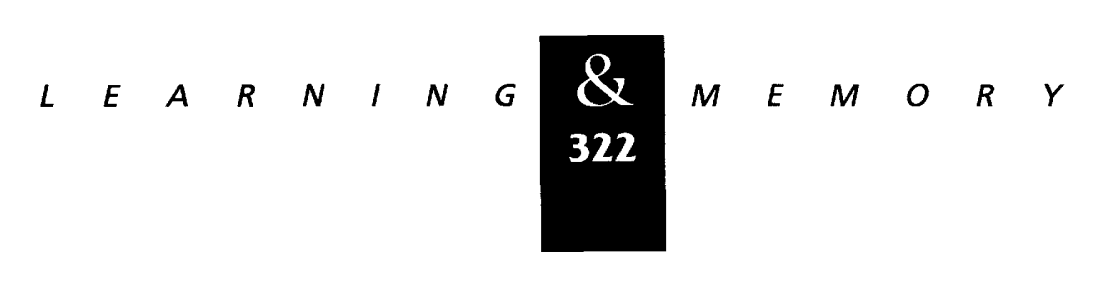


memories (Davis and Squire 1984; Alberini et al. 1995; Yin and Tully 1996). In addition, researchers have documented increases in gene expression in the rat central nervous system following behavioral training on a simple memory task (Tischmeyer et al. 1990; Nikolaev et al. 1992). Moreover, a recent study has shown that rats that have been exposed to sets of novel or highly familiar objects show an increase in the expression of the immediate-early gene c-fos. Interestingly, relatively high counts of c-fos expression were observed in the occipital cortex, area TE, and the perirhinal and entorhinal cortices (Zhu et al. 1995b). Because lesions of the hippocampal formation and perirhinal and entorhinal cortices collectively result in deficits on memory tasks that tax almost every sensory modality and because damage to these regions produce a comparable retrograde amnesia, it is possible, although highly speculative, that these brain regions may be involved in the modulation of protein biosynthesis or gene expression and that the initiation of these processes may underlie the formation of long-term memories.

In conclusion, the results of the present experiment indicate that in the rat, the perirhinal cortex and the fornix are involved in the consolidation of visual information. In addition, the results suggest that memory consolidation is a slow gradual process, which may remain active in the rat for a period of 3 to 4 weeks.

\section{Acknowledgments}

This work was supported by National Institutes of Health and the Charles A. Dana Foundation.

The publication costs of this article were defrayed in part by payment of page charges. This article must therefore be hereby marked "advertisement" in accordance with 18 USC section 1734 solely to indicate this fact.

\section{References}

Alberini, C.M., M. Ghirardi, Y.Y. Huang, P.V. Nguyen, and E.R. Kandel. 1995. A molecular switch for the consolidation of long term memory: cAMP-inducible gene expression. Ann. N.Y. Acad. Sci. 758: 261-286.

Alvarez, P. and L.R. Squire. 1994. Memory consolidation and the medial temporal lobe: A simple network model. Proc. Natl. Acad. Sci. 91: 7041-7045.

Bunsey, M. and H. Eichenbaum. 1993. Critical role of the parahippocampal region for paired-associate learning in rats. Behav. Neurosci. 107: 740-747.

Burwell, R., M. Witter, and D.G. Amaral. 1995. Perirhinal and postrhinal cortices of the rat: A review of the neuro- anatomical literature and comparison with findings from the monkey. Hippocampus 5: 390-408.

Cahill, L. and J.L. McGaugh. 1996. Modulation of memory storage. Curr. Opin. Neurobiol. 6: 237-242.

Cho, Y.H. and R.P. Kesner. 1996. Involvement of entorhinal cortex or parietal cortex in long term spatial discrimination memory in rats: Retrograde amnesia. Behav. Neurosci. 110: $436-442$.

Cho, Y.H., D. Beracochea, and R. Jaffard. 1993. Extended temporal gradient for the retrograde and anterograde amnesia produced by ibotenate entorhinal cortex lesions in mice. I. Neurosci. 13: 1759-1766.

Cho, Y.H., R.P. Kesner, and S. Brodale. 1995. Retrograde and anterograde amnesia for spatial discrimination in rats: Role of hippocampus, entorhinal cortex, and parietal cortex. Psychobiology 23: 185-194.

Corkin, S. 1984. Lasting consequences of bilateral medial temporal lobectomy: Clinical course and experimental findings in H.M. Semin. Neurol. 4: 249-259.

Davis, H.R. and L.R. Squire. 1984. Protein synthesis and memory: A review. Psychol. Rev. 96: 518-559.

Deacon, T.W., H. Eichenbaum, P. Rosenberg, and K.W. Eckmann. 1983. Afferent connections of the perirhinal cortex in the rat. J. Comp. Neurol. 220: 168-190.

Eacott, M.J., D. Gaffan, and E.A. Murray. 1994. Preserved recognition memory for small sets, and impaired stimulus identification for large sets, following rhinal cortex ablations in monkeys. Eur. J. Neurosci. 6: 1466-1478.

Eichenbaum, H., A. Fagan, and N.J. Cohen. 1986. Normal olfactory discrimination learning set and facilitation of reversal learning after medial-temporal damage in rats: Implications for an account of preserved learning abilities in amnesia. J. Neurosci. 6: 1876-1884.

Gaffan, D. 1994. Dissociated effects of perirhinal cortex ablation, fornix transection and amygdalectomy: Evidence for multiple memory systems in the primate temporal lobe. Exp. Brain Res. 99: 411-422.

Huppert, F.A. and M. Piercy. 1979. Normal and abnormal forgetting in organic amnesia: Effect of locus of lesion. Cortex 15: $385-390$.

Insausti, R., D.G. Amaral, and W.M. Cowan. 1987. The entorhinal cortex of the monkey. II. Cortical afferents. I. Comp. Neurol. 264: 356-395.

Kim, J.J. and M.S. Fanselow. 1992. Modality-specific retrograde amnesia of fear. Science 256: 675-677.

Kim, J.J., R.E. Clark, and R.F. Thompson. 1995. Hippocampectomy impairs the memory of recently, but not remotely, acquired trace eyeblink conditioned responses. Behav. Neurosci. 109: 195-203.

Levisohn, L.F. and O. Isacson. 1991. Excitotoxic lesions of the rat entorhinal cortex. Effects of selective neuronal damage on acquisition and retention of a non-spatial reference memory task. Brain Res. 564: 230-244.

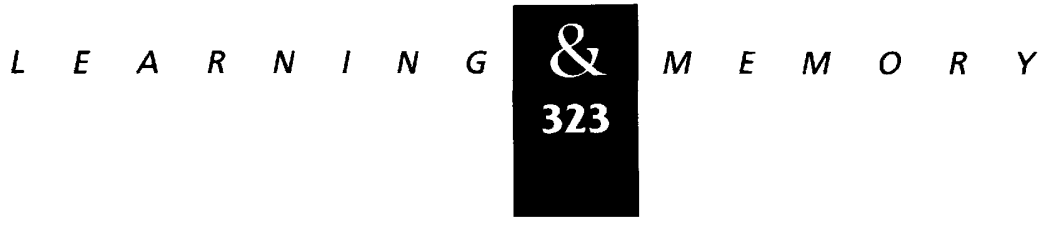


Matthies, H. 1989. Neurobiological aspects of learning and memory. Annu. Rev. Psychol. 40: 381-404.

McClelland, J.L., B.L. MCNaughton, and R.C. O'Reilly. 1995. Why are there complementary learning systems in the hippocampus and neocortex: Insights from the successes and failures of connectionist models of learning and memory.

Psychol. Rev. 102: 419-457.

McGaugh, J.L. and P.E. Gold. 1976. Modulation of memory by electrical stimulation of the brain. In Neural mechanisms of learning and memory (ed. M. Rosenzweig and E.L. Bennet), pp. 549-560. MIT Press, Cambridge, MA.

Meunier, M., \}. Bachevalier, M. Mishkin, and E.A. Murray. 1993. Effects on visual recognition of combined and separate ablations of the entorhinal and perirhinal cortex in monkeys. J. Neurosci. 13: 5418-5432.

Morris, R.G.M., P. Garrud, J.N.P. Rawlins, and J. O'Keefe. 1982. Place navigation impaired in rats with hippocampal lesions. Nature 297: 681-683.

Mumby, D.G. and J.P. Pinel. 1994. Rhinal cortex lesions and object recognition in rats. Behav. Neurosci.

108: $321-326$.

Mumby, D.G., J.P. Pinel, and E.R. Wood. 1990. Nonrecurring items delayed nonmatching-to-sample in rats: $A$ new paradigm for testing nonspatial working memory. Psychobiology 18: 321-326.

Murray, E.A., E.A. Gaffan, and R.W. Flint. 1996. Anterior rhinal cortex and amygdala: Dissociation of their contributions to memory and food preference in rhesus monkeys. Behav. Neurosci. 110: 30-42.

Myhrer, T. and K. Wangen. 1996. Marked retrograde and anterograde amnesia of a visual discrimination task in rats with selective lesions of the perirhinal cortex. Neurobiol. Learn. Mem. 65: 244-252.

Nikolaev, E., B. Kaminska, W. Tischmeyer, H. Matthies, and L. Kaczmarek. 1992. Induction of expression of genes encoding transcription factors in the rat brain elicited by behavioral training. Brain Res. Bull. 28: 479-484.

Otto, T. and H. Eichenbaum. 1992. Complementary roles of the orbito-frontal cortex and the perirhinal-entorhinal cortices in an odor-guided delayed non-matching to sample task. Behav. Neurosci. 5: 762-775.

Paxinos, G. and C. Watson. 1986. The rat brain in stereotaxic coordinates. Academic Press, San Diego, CA.

Salmon, D.P., S. Zola-Morgan, and L.R. Squire. 1987. Retrograde amnesia following combined hippocampus-amygdala lesions in monkeys. Psychobiology 15: $37-47$.

Saper, C.B. 1985. Organization of cerebral cortical afferent systems in the rat. II. Hypothalamocortical projections. J. Comp. Neurol. 237: 21-46.
Squire, L.R. 1981. Two forms of human amnesia: An analysis of forgetting. J. Neurosci. 1: 635-640.

1987. Memory and brain. Oxford University Press, New York, NY.

Squire, L.R. and C.W. Spanis. 1984. Long gradient of retrograde amnesia in mice: Continuity with the findings in humans. Behav. Neurosci. 98: 345-348.

Squire, L.R., P.C. Slater, and P.M. Chase. 1975. Retrograde amnesia: Temporal gradient in very long term memory following electroconvulsive therapy. Science 187: 77-79.

Staubli, U., D. Fraser, M. Kessler, and G. Lynch. 1986. Studies on retrograde and anterograde amnesia of olfactory memory after denervation of the hippocampus by entorhinal cortex lesions. Behav. Neural Biol. 46: 432-444.

Suzuki, W.A., S. Zola-Morgan, L.R. Squire, and D.G. Amaral. 1993. Lesions of the perirhinal and parahippocampal cortices in the monkey produce long-lasting memory impairment in the visual and tactual modalities. J. Neurosci. 13: $2430-2451$.

Swanson, L.W. and W.M. Cowan. 1979. The connections of the septal region in the rat. J. Comp. Neurol. 186: 621-656.

Tischmeyer, W., L. Kaczmarek, M. Strauss, R. Jork, and H. Matthies. 1990. Accumulation of c-fos mRNA in rat hippocampus during acquisition of a brightness discrimination. Behav. Neural Biol. 54: 165-171.

Vnek, N., T.G. Gleason, L.F. Kromer, and L.A. Rothblat. 1995. Entorhinal-hippocampal connections and object memory in the rat: Acquisition versus retention. J. Neurosci. 15: 3193-3199.

Wiig, K.A. and D.K. Bilkey. 1994a. The effects of perirhinal cortical lesions on spatial reference memory in the rat. Behav. Brain Res. 63: 101-109.

1994b. Perirhinal cortex lesions in rats disrupt performance in a spatial DNMS task. NeuroReport 5: 1405-1408.

1995. Lesions of rat perirhinal cortex exacerbate the memory deficit observed following damage to the fimbria-fornix. Behav. Neurosci. 109: 620-630.

Wincour, G. 1990. Anterograde and retrograde amnesia in rats with dorsal hippocampal or dorsomedial thalamic lesions. Behav. Brain Res. 38: 145-154.

Yin, J.C.P. and T. Tully. 1996. CREB and the formation of long term memory. Curr. Opin. Neurobiol. 6: 264-268.

Zhu, X.O. and M.W. Brown. 1995. Changes in neuronal activity related to the repetition and relative familiarity of visual stimuli in rhinal and adjacent cortex of the anaesthetised rat. Brain Res. 689: 101-110. 
Zhu, X.O., M.W. Brown, and J.P. Aggleton. 1995a. Neuronal signaling of information important to visual recognition memory in rat rhinal and neighboring cortices. Eur. J. Neurosci. 7: 753-765.

Zhu, X.O., M.W. Brown, B.J. McCabe, and J.P. Aggleton. $1995 \mathrm{~b}$. Effects of the novelty or familiarity of visual stimuli on the expression of the immediate early gene c-fos in the rat brain. Neurosci. 69: 821-829.

Zilles, K. 1990. Anatomy of the neocortex: Cytoarchitecture and myeloarchitecture. In The cerebral cortex of the rat (ed. B. Kolb and R.C. Tees), pp. 77-112. MIT Press, Cambridge, MA.

Zola-Morgan, S. and L.R. Squire. 1990. The primate hippocampal formation: Evidence for a time-limited role in memory storage. Science 250: 288-290.

Zola-Morgan, S., L.R. Squire, D.G. Amaral, and W.A. Suzuki. 1989. Lesions of the perirhinal and parahippocampal cortex that spare the amygdala and the hippocampal formation produce severe memory impairment. /. Neurosci. 9: $4335-4370$.

Received September 5, 1996; accepted in revised form November 11, 1996. 


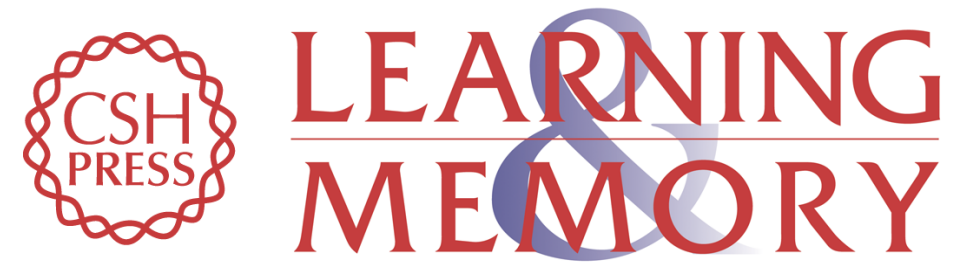

\section{Temporally graded retrograde amnesia following separate and combined lesions of the perirhinal cortex and fornix in the rat.}

K A Wiig, L N Cooper and M F Bear

Learn. Mem. 1996, 3:

Access the most recent version at doi:10.1101//m.3.4.313

References This article cites 49 articles, 10 of which can be accessed free at: http://learnmem.cshlp.org/content/3/4/313.full.html\#ref-list-1

License

Email Alerting Receive free email alerts when new articles cite this article - sign up in the box at the Service top right corner of the article or click here. 